\title{
Universalismo generalista y particularismo moderado. Réplica a mis críticos*
}

\author{
General Universalism and Moderated \\ Particularism. Reply to My Critics
}

\author{
René González de la Vega
}

No es contra el mundo que embiste el rinoceronte sino contra esos cuernos que por nada se quitan de en medio de sus ojos

Jorge Luján*

Recepción y evaluación de propuesta: 20/2/2014

Aceptación: 20/03/2014

Recepción y aceptación final: 22/6/2015

Resumen: El texto responde a las críticas realizadas por Fernando Lizárraga, Julio Montero y Graciela Vidiella en esta misma revista al artículo "Legalismo moral y tolerancia. Crónica de una muerte anunciada". Palabras clave: liberalismo, tolerancia, conflictos prácticos.

\begin{abstract}
The article answers to the critics advanced by Fernando Lizárraga, Julio Montero y Graciela Vidiella in this review to the essay "Moral legalism and tolarance. Chronicle of a Death Foretold".
\end{abstract}

Key words: liberalism, tolerance, practical conflicts.

* Quisiera agradecer a la Revista Discusiones y en particular a Hernán Bouvier por la posibilidad de participar en este número participar. También quisiera agradecer a Guillermo Lariguet y a Rafael Caballero por su apoyo y por los comentarios y correcciones que hicieron a una versión preliminar de este trabajo.

${ }^{* *}$ Luján, J.,, Palabras manzana, Aique, Buenos Aires, Argentina, 2010, pág. 14 


\section{I.}

Puede sonar extraño continuar una discusión diciendo que coincido con quienes me han criticado y con quienes han hecho observaciones contrarias a lo que he dicho antes. De ser así, por qué no mejor quedarme callado y asentir. Por qué no mejor aceptar el error y continuar el camino.

De hacer esto parecería que claudico frente a lo antes dicho, o que nunca me tomé demasiado en serio las cosas que me dijeron. Pero nada más lejano que eso. Me he tomado muy en serio lo que me han dicho y, de hecho, sostengo muchas de las cosas que ellos dicen. Hasta cierto grado me identifico con algunas de sus posturas y argumentos. Pero también es verdad que no claudicaré en mi postura, y trataré de insistir en ella. Trataré de persuadirlos una vez más, aunque con distintos argumentos.

Los tres trabajos que anteceden a este, toman caminos cercanos aunque no idénticos. Los trabajos de Montero y Lizárraga centran su atención exclusivamente en una versión específica del liberalismo deontológico: el liberalismo político. Esto, sin duda, reduce la circunferencia de discusión a una clase específica, bastante particular y peculiar (dentro de la larga tradición liberal) del liberalismo deontológico. Aún siendo así, debo reconocer que ambos autores han dado en el blanco de una distinción que se debe hacer explícita y que he tratado de denunciar en mi texto anterior, y que ahora abordaré con mayor detalle.

Fernando Lizárraga ha estructurado su trabajo sobre la distinción entre el ámbito público (político) y el privado (personal). En ese sentido, Fernando argumenta a partir de la idea de que la tolerancia, para Rawls, es fundamentalmente una virtud institucional y no personal. Efectivamente, como argumenta Lizárraga, el propósito de la teoría de Rawls es el de generar una sociedad plural estable y para esto le basta el "consenso traslapado". En ese sentido, la tolerancia no resultaría ser la "promesa normativa" mediante la cual las instituciones públicas podrían lograr una estabilidad social. Para eso basta con aceptar los principios derivados de un consenso profundo o traslapa- 
do. Esto, en términos menos rawlsianos, significaría que no está en las instituciones públicas el tolerar específicas concepciones del bien, sino el asegurar la aceptación y el respeto de los derechos derivados de ese consenso. A partir de esta estructura, Lizárraga concluye que la desaparición de la tolerancia no sería algo sorpresivo pues, en realidad, "!es precisamente lo que hace Rawls!, ya que la tolerancia es fundamentalmente una característica de las instituciones bajo un equilibrio de fuerzas que él denomina modus vivendi, una instancia previa e inferior al deseable consenso traslapado donde ya no se requiere tolerancia".

Salvo en algunas cuestiones de detalle, en términos generales, estoy de acuerdo en la reconstrucción general que hace Fernando de la obra de Rawls. Sin embargo, consideremos lo siguiente: si la división, marcada por Lizárraga a través de Rawls, le elimina toda razón de ser a la tolerancia en el ámbito público debido a la existencia del consenso traslapado (con la cual coincido, aunque por distintas razones: efectivamente, cuando esta clase de liberalismo habla de derechos, la tolerancia se vuelve irrelevante), aún seguiría estando pendiente el asunto relativo a la tolerancia en el ámbito privado. Definitivamente, como sostiene Fernando, Rawls no se encarga de ello y poca atención le presta al asunto. Sin embargo, esta clase de respuestas no me satisfacen. Nos invitan a pensar que el liberalismo deontológico es únicamente una doctrina que se encarga de diseñar una arquitectura institucional artificial, pues no es una doctrina que pueda ser adoptada con convicción y certeza por los individuos para guiar sus vidas; individuos que además serán los que al final del día se encarguen de dirigir dichas instituciones.

Pero aún siendo así, concediendo que en el ámbito público la tolerancia no es necesaria, mi tesis ha sido que el liberalismo deontológico (político o comprensivo) tiene que dar respuesta a los problemas de tolerancia en el ámbito privado. Por ello, decía que el problema que me interesa es el de "un solo agente" y no el de "varios agentes"; es en esos casos donde me parece que la estructura deontológica del razonamiento práctico tensiona aún más con los requerimientos de la tolerancia. Sobre todo, como ya he dicho, porque la distinción entre 
tolerancia pública y tolerancia privada me parece tan artificial como la idea de una moral plenamente independiente de la ética ${ }^{1}$.

A pesar de algunas coincidencias, la respuesta de Julio Montero no coincide con Lizárraga en un aspecto. El propósito de Julio es el de mostrar que me equivoco en el sentido de que el liberalismo deontológico no solo puede acomodar la virtud de la tolerancia, sino que además puede darle una preponderancia que ninguna otra variante del liberalismo le concedería. Para Montero, la tolerancia es posible y plenamente practicable bajo el esquema del liberalismo político, aunque no bajo otro esquema de liberalismo deontológico como son los llamados liberalismos "comprensivos" o "éticos". Para Montero, la tolerancia podría desdibujarse si adoptamos variantes más integradas del liberalismo que abandonan la esquizofrenia entre lo público y lo privado.

Para Montero, como para Lizárraga, la tolerancia es posible bajo el esquema del liberalismo político dado que este distingue entre dos contextos distintos de justificación: el público y el privado; el moral y el ético. Pero también nos dice, al igual que Lizárraga, que en realidad, para el liberalismo político prescindir de la tolerancia no sería un problema. Fundamentalmente, porque este ya supone un compromiso con el derecho de toda persona a vivir de acuerdo con su propia concepción del bien aunque otros la consideren errada.

He tratado de argumentar, anteriormente, que la distinción entre diferentes contextos de justificación no es una característica propia o exclusiva del liberalismo político, en particular, sino del liberalismo deontológico, en general; una cosa es distinguir entre 'moral' y 'ética', y otra cosa muy distinta es sostener la independencia de la 'moral' frente a la 'ética'. En ese sentido, de hecho he sostenido que el liberalismo político puede prescindir de la tolerancia en el ámbito público debido al "consenso traslapado". Pero aún así, habría un

1 Sin duda alguna, la distinción entre ambas clases de tolerancia guarda cierta relevancia desde un punto de vista analítico y filosófico; sirve para explicar ciertos problemas y distinguir entre diferentes formas de solución. Sin embargo, la distinción se desdibuja en la cotidianeidad de nuestras vidas. La diferencia entre distintos ámbitos no es tan tajante, ni tan sencilla de percibir. 
tema que debe solucionar y es el relacionado con los conflictos que he llamado "profundos"; conflictos entre principios morales, que pertenecen a dicho ámbito.

Sin embargo, esto solo lo permite la pureza del ámbito moral lograda a través del criterio de independencia — freestanding —, que aún estando libre de contaminaciones "éticas" tendría problemas propios de su ámbito, como he señalado. Pero incluso aceptando la posibilidad de que exista una moral freestanding hay que tener en mente un punto que el mismo Rawls contempla. Este ámbito, el ámbito de lo razonable, está en constante contacto con el ámbito de lo racional, con el ámbito de nuestras concepciones del bien. En ese sentido, el conflicto arranca cuando adoptamos convicciones particulares que no coinciden con los principios morales, o cuando dos convicciones de una misma concepción del bien, dadas ciertas circunstancias específicas de cada caso, resultan ser incompatibles. Es en esos casos donde encontramos otra clase de problemas (que he denominado "poco profundos"). Es decir, aún aceptando que el liberalismo deontológico no requiere de la tolerancia en el ámbito público, la pregunta sigue persistiendo cuando nos referimos a los problemas de un solo agente; a los conflictos en el ámbito privado. Si en estos casos también argumentamos que esa clase de problemas no es el interés del liberalismo deontológico, luego entonces, se confirmaría la tesis de que la tolerancia frente a esta clase de liberalismo es, en el mejor de los casos, innecesaria.

Por ello me parece que es esta clase de problemas los que le interesan a Graciela Vidiella. Esa clase de problemas que una persona, liberal, debe resolver en la cotidianeidad de su vida. Cuando los criterios de la moral son los que terminan derrotando, de una vez y para siempre, alguna de nuestras convicciones, es cuando existe una imposición de la moral y la tolerancia se torna inservible. En realidad, esta clase de conflictos, como afirma Graciela en su trabajo,

requiere de la sensibilidad moral para captar el contexto en el que correspondería aplicarla; requiere que se tenga registro de las personas involucradas, que se comprenda cuál es el fin de 
la acción que corresponde llevar a cabo y que se conozcan los medios para lograrlo.

En ese mismo sentido, Julio está consciente de que se requiere una deliberación que sopese numerosas variables en un análisis caso por caso. Algunas de esas variables se relacionan con hechos circunstanciales, otras con contextos sociales particulares, y otras suponen una consideración de las consecuencias que diversos cursos de acción podrían producir.

Pero como sabemos, ninguna de estas cosas es posible si adoptamos todos y cada uno de los criterios del deontologismo liberal, pues aspectos relacionados con la "sensibilidad moral" o la "importancia de los contextos" son incompatibles con ellos.

Este, me parece, es el problema en el que todos convergen. Guardo la sospecha, aunque puede estar equivocada, de que esta es la preocupación central vertida en los tres trabajos que me critican. Por ejemplo, Vidiella afirma que "la pregunta socrática es tan importante como la kantiana", expresando esta preocupación, y del mismo modo cuando Montero habla de la "esquizofrenia propia del liberalismo deontológico" y del "desgarramiento que se produce al abstenernos de actuar con base en convicciones que seguimos considerando verdaderas", está dando cuenta de esta misma clase de problemas, de la misma forma que Lizárraga, cuando afirma que en realidad lo que Rawls quiere es "superar la tolerancia por medio del consenso traslapado" y que ve "la necesidad de que la tolerancia y otras virtudes sean no solo institucionales, como quiere Rawls, sino también personales". Hasta cierto grado, estas preocupaciones me indican que los tres filósofos están conscientes de que las decisiones éticas se encuentran un tanto asfixiadas por el mundo de la moral.

Mi sospecha es que mis tres interlocutores guardan la misma preocupación o, al menos, son conscientes de la posibilidad de que la moral, entendida en términos deontológicos, termina en algunos casos asfixiando a la ética. Puede ser que esta clase de sospechas hayan sido las que empujaron a un autor como Bernard Williams a decir 
que si concebimos a la moral de esa manera "estaríamos mucho mejor sin ella" 2 .

Ante esta situación he luchado con la tentación de referirme a cada uno de los textos y contestar a cuestiones muy particulares. Cuestiones de interpretación o de apreciación sobre lo que dicen autores específicos. Leyendo los textos de mis interlocutores, tengo la impresión de que los tres me están exigiendo mucho más que eso. Es decir, para ellos esto significaría que me estoy saliendo por la tangente, o sea, optar por la vía fácil de la refutación específica y dejar pendiente el reclamo central que me han hecho: dar una respuesta que, al menos de manera tentativa, trate de tomarse "en serio tanto la pregunta kantiana como la socrática"; que diga cómo evitar la "esquizofrenia" o "como conjugar la virtud de la tolerancia en el ámbito personal, dentro del marco del liberalismo deontológico".

Estas consideraciones me ponen un reto en frente: contestar la pregunta que los tres, explícita e implícitamente, formulan en sus trabajos. En lo que sigue trataré de articular una respuesta que en adelante llamaré la respuesta contextual, la que me parece puede aportar las coordenadas suficientes para armonizar la tolerancia con ciertos requisitos normativos del liberalismo deontológico, aunque no con todos. Esta respuesta procurará aportar una solución para los conflictos de tolerancia en el ámbito privado sin tener que abandonar el liberalismo deontológico in toto.

Al final de este trabajo, dedicaré dos párrafos para explicar de manera general en qué consiste mí crítica al liberalismo deontológico, esperando que estos ayuden al lector a comprender mi crítica inicial, mi respuesta y mi postura filosófica frente al liberalismo en general.

Ahora bien, no diré nada sin antes hacer explícito mi agradecimiento profundo a los tres filósofos que se han tomado el tiempo de responder y criticar mis ideas. En ocasiones uno tiende a perder el rumbo y comienza a cuestionarse sobre el valor de lo dicho y los propósitos de lo hecho. Pero también, hay ocasiones en los que algunos

2 Williams, B., Ethics and the Limits of Philosophy, Cambridge, Harvard University Press, 1985, pág. 174 
tenemos la fortuna de encontrarnos con personas como Lizárraga, Montero y Vidiella que nos recuerdan el valor de lo dicho, el placer de lo hecho y sobre los resultados concretos de nuestros esfuerzos.

No puedo más que reconocer y agradecer en estas primeras líneas, la deferencia de trato, la discusión abierta, sincera y franca que me han ofrecido estos tres grandes filósofos. Pero sobre todo, agradecerles por acorralar mis argumentos al punto de tener que repensar muchos de ellos, abandonar algunos y reformular otros. Agradezco a estos tres profesores, personas de altas capacidades intelectuales y personales, porque con sus palabras han revitalizado mi interés por seguir discutiendo estos temas.

\section{II.}

Insistiré en que la fuente de tensiones entre la tolerancia y el liberalismo deontológico es el universalismo moral. Soy consciente que al decir esto no resuelvo mucho el tema. El universalismo moral puede entenderse, al menos, desde dos perspectivas distintas. Digamos que una de estas perspectivas deriva directamente del pensamiento de Kant y, la otra, fundamentalmente, de autores como Richard Hare. La primera clase de tesis universalista, la kantiana, dicta que una persona debe actuar según la máxima de que sus actos se conviertan en ley universal. Es decir, lo que viene a decirnos Kant es que para que mis acciones sean consideradas correctas tienen que ser consideradas de ese modo por cualquier agente racional ${ }^{3}$. Esta podría entenderse como una universalidad de la imparcialidad. Diferente de aquella que es defendida por autores como Hare (entre otros). Esta otra clase de universalismo moral sostiene que si un agente decide ' $X$ ' en la circunstancia ' $\mathrm{C} 1$ ', tiene necesariamente que decidir también ' $\mathrm{X}$ ' en un caso 'C2' que guarde las mismas características relevantes contenidas

3 Kant, I., Groundwork of the Metaphysics of Morals, Cambridge University Press, 1997, págs. 40 y ss. Véase, también, Carlos S. Nino, Ética y Derechos Humanos. Un Ensayo de Fundamentación, Buenos Aires, Astrea, 2005, págs.110-111 
en ' $\mathrm{C} 1$ ' . Esta clase de universalidad podríamos denominarla como una universalidad generalista. Cuando he atacado al universalismo es porque este no permite la posibilidad de que se tolere un acto ' $A$ ' de un agente ' $\mathrm{X}$ ' y después no tolere un acto ' $\mathrm{A}$ ' de un agente ' $\mathrm{Y}$ '. Esta forma de universalismo generalista es la que tensiona directamente con la tolerancia y, por ello, es a la que atacaré ahora.

Esta clase de universalismo es merecedora, fundamentalmente, de dos críticas. La primera de ellas es una crítica en la que he venido insistiendo. En casos de tolerancia, la tesis de consistencia que el universalismo moral impone sobre el razonamiento práctico repercute indiscutiblemente en la configuración de los sistemas normativos. Como había argumentado anteriormente, el resultado de esta doctrina es que si una persona tolera una conducta determinada debe incluir dicha conducta dentro de su catálogo de conductas permitidas. Si no la tolera, en cambio, la incluye dentro de su catálogo de conductas prohibidas. Es decir, el agente de tolerancia después de tolerar, por razones lógicas, tiene que deshacerse o reafirmar la convicción lesionada. Esta forma de configurar la tolerancia, la lleva a una conclusión que me parece contraintuitiva: cada acto de tolerancia es un paso hacia la intolerancia. Las convicciones que pueden llegar a sostener un individuo son finitas. Sin duda. Si cada acto de tolerancia es irrepetible, ya que debido a los criterios de consistencia (impuestos por esta clase de universalidad generalista) la convicción en juego es derogada o confirmada, cualquier otra conducta que sea subsumible en dicha norma ya no podrá ser tolerable. La tolerancia, en tal caso, por razones lógicas se vuelve suicida.

Subrayo el hecho de que esto sucede por razones lógicas, ya que se podría contraargumentar que la tolerancia debe tener límites y que no es moralmente aceptable tolerarlo todo. Efectivamente, estoy de acuerdo con eso. Sin embargo, el ideal sería que los límites de la tolerancia sean impuestos por razones morales y no, únicamente, por criterios lógicos.

4 Hare, R. H., Freedom and Reason, Oxford, Clarendon Press, 1964, Cap. 2 
A grandes rasgos eso es lo que he defendido hasta ahora. Ahora bien, en anterior texto he defendido una tesis que sostiene la dependencia contextual de la tolerancia, esta tesis será la que ahora me permitirá desarrollar lo que llamaré: La respuesta contextual.

La tesis que defiende el hecho de que la tolerancia es contextualmente dependiente se enfrenta, en realidad, con un tema escabroso de la filosofía moral como lo es el de los límites de la tolerancia. Pero se enfrenta a él desde una perspectiva distinta a la que el liberalismo deontológico tradicionalmente ha defendido. En vez de considerar un catálogo de conductas determinadas y de actos que a priori son considerados como intolerables, la respuesta contextual, si bien también considera que no todo es tolerable, sostiene que para saber qué es tolerable y qué no lo es debe ser definido, entre otras cosas, por las características relevantes ofrecidas por cada caso en concreto. En este sentido, la respuesta contextual no abandona la idea de actos intolerables, aunque sí abandona la idea de que exista un catálogo de conductas y acciones que son previamente consideradas intolerables.

Según esta perspectiva, los límites de la tolerancia son impuestos por la relevancia que pueden adquirir distintas razones morales dependiendo de los contextos o de las circunstancias específicas de la tolerancia. Por lo tanto, esta perspectiva rechaza la tesis deontológica según la cual "las consideraciones morales están determinadas para la eternidad y son perfectamente precisas y consistentes" .

La respuesta contextual está relacionada con algunas de las objeciones que ha hecho Jonathan Dancy en su Moral Reasons a la doctrina del universalismo moral, fundamentalmente, con su crítica a las posturas universalistas (generalistas) como posturas negligentes ante el valor de las características sobresalientes aportadas por cada contexto. La respuesta contextual a los casos de tolerancia acepta la existencia de reglas y principios, pero también defiende que normalmente en cuestiones de carácter práctico actuar de una forma razonable e inteligente implica hacer algo más que obedecer de manera pasiva y ciega

5 Wallace, J. D., Moral Relevance and Moral Conflict, Ithaca, New York, Cornell University Press, 1988, pág. 53 
Universalismo generalista y particularismo moderado. Réplica a mis críticos

lo que ciertas reglas nos dicen que hagamos ${ }^{6}$. Wallace ha resumido brillantemente esta idea de la siguiente forma:

Hablando en términos prácticos, a menos que queramos mantener nuestra insensatez debemos aprender al mismo tiempo a adaptarnos a las circunstancias que cambian de manera indefinida frente a las circunstancias en las que nos enseñaron [a actuar][...]

Los individuos inteligentes aplican las reglas [dependiendo] de los conflictos que los acosan ${ }^{7}$.

Pero para concluir con la digresión, primero presentaré los argumentos de Dancy con los que la respuesta contextual es compatible y, más tarde, explicaré la clase de particularismo que es adoptado por la respuesta contextual, el cual se aleja considerablemente del particularismo defendido por Dancy, aunque recoge partes de él.

Uno de los aspectos con los que coincido plenamente con Dancy es que la mejor cualidad del deontologismo, al final, resulta ser uno de sus peores vicios. Dancy dice que:

La virtud técnica de esta postura es que da un buen sentido a la idea de los principios morales, que si son verdaderos, entonces, son necesariamente verdaderos. Tradicionalmente, esta idea acumula tres cosas. La primera es la inservible idea de que si una acción es correcta, luego entonces, toda acción que sea similar en sus aspectos no-morales a ella también debe ser correcta [...] La segunda idea no es inserviblemente verdadera, pero grotescamente falsa. Esta se refiere a la idea de que si una acción es correcta por sus características 'F-es', luego entonces, cualquier otra acción que contenga la calidad ' $F$ ' es necesariamente correcta [...] La tercera trata de que si una propiedad es moralmente relevante en un caso, luego entonces,

6 Ibídem, pág. 65

7 Ibidem, pág. 65. Los corchetes son míos. 
tendrá la misma clase de relevancia moral en cualquier otro caso en el que aparezca ${ }^{8}$.

Tal y como lo pone Dancy, parece que una de las debilidades del generalismo moral es que su esquema es tan rígidamente lógico que pone en duda la corrección moral de sus conclusiones prácticas (esta crítica iría de la mano con la apreciación de Graciela Vidiella cuando dice que esta reconstrucción corresponde mejor con sistemas jurídicos que con sistemas morales). Estas son algunas de las razones que he argumentado anteriormente de por qué esta postura, también, resulta lógicamente imposibilitada para hablar de tolerancia más de dos veces frente a una situación de similares características. Con este punto de partida, Dancy propone dos formas de crítica a esta clase de universalismo generalista. Una se refiere a los problemas internos que podemos encontrar dentro de esta clase de razonamiento y, la otra hace referencia al tipo de motivación que ofrece esta clase de universalismo moral.

Uno de los problemas centrales de esta noción del universalismo inicia, de acuerdo con Dancy, cuando "una persona que emite un juicio moral está comprometida con emitir el mismo juicio moral en cualquier otra situación con las mismas características relevantes"9. Existen dos clases de argumentos que atacan esta postura. El primero de ellos, que es al que Dancy presta más atención en su libro, está dirigido a la estructura interna del universalismo. El segundo, que ha sido desarrollado con mucha mayor precisión por John McDowell, intenta minar los criterios de motivación que pueda tener esta propuesta ${ }^{10}$. Es verdad que ambos argumentos a pesar de haber sido presentados como dos argumentos distintos, hasta cierto grado, se solapan; logran ser consistentes. Sin embargo, para los propósitos que tengo en mente solo me voy a concentrar en el argumento sobre los problemas internos de Dancy.

Dancy comienza su argumento con una demoledora afirmación:

8 Dancy, J., Moral Reasons, Blackwell, Oxford \& Cambridge, 1993, pág. 69.

9 Ibídem, pág. 80.

${ }^{10}$ McDowell, J:, "Non-cognitivism and Rule-following", en Ted Honderich (ed.) Morality and Objectivity: A Tribute to J. L. Mackie, London, Routledge and Kegan Paul, 1985, págs. 110-29. 
"la doctrina del universalismo es claramente falsa"11. Por supuesto, esta clase de afirmaciones requieren de un sustento fuerte; no basta con la calificación de falsa para que en realidad lo sea. Sobre todo, cuando, como he dicho, la filosofía moral occidental ha estado regida por la doctrina de la universalidad desde hace bastante tiempo. Dancy soporta su afirmación diciendo, en primer lugar, que

no es posible que uno esté obligado a emitir el mismo juicio donde sea que se presenten el limitado grupo de propiedades recurrentes. Ya que en un nuevo caso puede haber fuertes razones en contra del juicio que no estuvieron presentes en el primer $\mathrm{caso}^{12}$.

A esta clase de razones Dancy las llama "derrotadores" (defeaters). No necesariamente se requiere que los "derrotadores" estén ausentes en el primer caso. Por el contrario, pudieron haber estado presentes en el primer caso también, pero con mucha menor intensidad que como lo hacen en el segundo caso. Dancy explica esto con el siguiente ejemplo, bastante esclarecedor a mi juicio:

Una primera acción es generosa y amable aunque un poco tosca. Nosotros la aprobamos por su generosidad y amabilidad, no por su tosquedad. Una segunda acción es tan amable y generosa como la primera, pero muy tosca. Ahora quisiéramos desaprobar dicha acción, pero la doctrina del universalismo, tal y como ha sido formulada, nos previene de hacer esto, al menos que revisemos nuestra opinión sobre la primera acción ${ }^{13}$.

Ciertamente, los universalistas, como afirma Dancy, en este punto pueden argumentar que en vez de prestar nuestra atención a las razones a favor de la primera acción (su generosidad y amabilidad) deberíamos prestar atención a todo el conjunto de razones, las que están a favor y las que están en contra de nuestra aceptación (la amabilidad,

${ }^{11}$ Dancy, J., op. cit., pág. 80.

${ }^{12}$ Ídem.

${ }^{13}$ Ídem. 
la generosidad y la tosquedad). Sin embargo, Dancy replica a esta estrategia del universalismo con este otro contraejemplo:

Supongamos que un hombre golpea con su coche a una mujer y, por ello, la lleva a un hospital. Cuando él paga las cuentas, la visita recurrentemente y pone especial atención a su condición médica, aprobamos sus acciones. Puesto que son expresiones de arrepentimiento y un intento por enmendar hasta cierto grado lo sucedido. Estos hechos nos sirven de razones para aprobar sus actos. Pero no nos comprometen a aprobar los actos de una persona que se conduce de la misma manera, pero que sus propósitos finales son los de seducir a la mujer y alejarla de su esposo. Y no podemos escapar de este punto diciendo que entre nuestras razones para aprobar la conducta de la primera persona estaba también el hecho de que él no tenía en mente este propósito ${ }^{14}$.

Ante esto, los defensores del universalismo generalista, dice Dancy, estarán tentados a ampliar aún más la base universalista. Ahora requerirán similitudes no solo respecto a todos los factores que estuvieron a favor y en contra del juicio original, sino también con respecto a la presencia o ausencia de ciertos factores que pudieran haber afectado en algún grado el juicio moral original. Por supuesto, lo que está sucediendo en este momento es que la base de la doctrina universalista está comenzando a expandirse de una manera en la que originalmente nunca lo había hecho. Esto acarreará la eventualidad de que en cualquier momento el universalismo claudicará al tener que incluir, dentro de su base de criterios relevantes de los casos, todas las propiedades naturales que incluye cada caso particular, perdiendo, por consiguiente, la estructura que hasta ahora habían mantenido.

Esta situación presentada por los argumentos de Dancy me parece esclarecedora. Por un lado, como he venido repitiendo, uno de los problemas más grandes que presentan los agentes de tolerancia es el de asumir el compromiso de consistencia que el universalismo les impone. Pero al mismo tiempo, los agentes de tolerancia que seguían 
inmiscuidos en las perspectivas morales que son propias del universalismo sufrían una especie de vértigo moral cada vez que se les presentaba la posibilidad de no asumir las tesis de consistencia universalista y pensar que podría llegar a ser correcto tolerar una acción ' $\mathrm{X}$ ' hoy y no tolerar una acción ' $\mathrm{X} 1$ ' — que compartía las mismas características relevantes- mañana ${ }^{15}$. Esta situación, como lo expuse anteriormente, los orillaba a tener que derogar alguna de sus convicciones morales.

Además, de acuerdo con las consecuencias prácticas de las tesis del universalismo, la derogación de la convicción no significa una pérdida o un sacrificio moral. Por el contrario, el haber derogado la convicción moral implica el resultado de una deliberación que encontraba que dicha convicción era falsa. Ahora no me detendré mucho en esta situación de los sentimientos de pérdida o sacrificio que pueden generar los actos de tolerancia, pues lo haré más adelante. Por el momento, me detendré en la sospecha que guardo ante la doctrina del universalismo generalista y que ha dado pie a mi crítica entera en el artículo anterior.

Me parece que las críticas de Dancy, si son bien atendidas, deben librarnos de la perplejidad teórica en la que estábamos sumergidos con los ejemplos de la madre 'culta' de Graciela Vidiella y con el del profesor universitario. Estos problemas, bajo el prisma teórico de la moral universalista, se veían mucho más trágicos de lo que llegan a verse ahora.

Antes de entrar al punto que me interesa destacar aquí, debo mencionar que el particularismo defendido por Dancy no solo se dedica a dar un embate al universalismo basado en contraejemplos. La defensa de Dancy sobre el particularismo moral es un tanto más profunda que el simple hecho de encontrar ejemplos ingeniosos que pongan al

${ }^{15}$ Recuérdese que la idea del vértigo moral fue explicado en el texto anterior. Ahí decía que es uno de los argumentos que presenta McDowell, y que Dancy también apoya. Este argumento consiste en la sensación de vértigo que puede sufrir un agente cuando se ve alejado de la consistencia universalista. La sensación de vértigo en este caso estaría referida a la situación propia de alguien que piensa que está a punto de caer a un precipicio y no tiene de dónde agarrarse. En ese sentido, aceptada la analogía, el barandal de los partidarios de la doctrina universalista serían los principios generales, y su resistencia ante el vacío, la consistencia. 
universalismo en aprietos. Podemos decir que la crítica de Dancy al universalismo corre por tres distintas vías. Dancy critica tres figuras propias de las teorías universalistas generalistas: la concepción atomista de las razones morales, la relación de superveniencia y el modelo subsuntivo. En lo que sigue, solo me centraré en las críticas a la concepción atomista de las razones morales y del modelo subsuntivo, ya que son las dos características que generan el mayor número de problemas para el ideal moral de la tolerancia.

Dancy critica la concepción atomista de las teorías universalistas (generalistas) diciendo que uno de los problemas del universalismo se encuentra en la forma en cómo conciben el papel que juegan las razones morales dentro del razonamiento práctico. Los universalistas piensan que toda situación puede describirse en términos de una combinación de elementos independientes (atómicos) e irreductibles. Por ejemplo, una norma moral o un principio pueden estipular como propiedades relevantes ' $a$ ', 'b', 'c'y ' $d$ ', para determinar la incorrección moral de un comportamiento dado. Ahora supongamos que en un comportamiento solo están presentes las propiedades ' $a$ ' $y$ ' $d$ ', por lo tanto, su presencia es suficiente para que dicho comportamiento sea considerado moralmente incorrecto según los criterios lógicos del universalismo.

El particularismo de Dancy, en cambio, sostiene una concepción holista de las razones morales ${ }^{16}$. La relevancia moral de cada una de las propiedades que aparecen dentro de un caso es dada por las circunstancias y el contexto determinado en el que dichas propiedades hayan tenido lugar. Martínez Zorrilla lo explica de la siguiente manera:

Supongamos que a significa comunicar información falsa (mentir). En una concepción universalista, que una acción individual sea subsumible en $a$ constituye siempre (por razones lógicas) una razón moral (un elemento moralmente relevante), precisamente porque es tenido en cuenta por una norma del sistema. En cambio, para el particularismo, que el mentir tenga relevancia moral (independientemente de que esta sea a favor o en contra de la corrección del comportamiento) depende del contexto 
Universalismo generalista y particularismo moderado. Réplica a mis críticos

en el que se sitúa, y por tanto de los muchos otros elementos también presentes en cada situación concreta ${ }^{17}$.

Aceptar que la relevancia moral de las propiedades es otorgada por las circunstancias y el contexto en el que se encuentran es de suma importancia para el tema de la tolerancia. Antes de entrar en esto, explicaré la crítica que hace Dancy al modelo subsuntivo. Esta es otra crítica que me parece central del particularismo de Dancy. Claro, la crítica que hace a la concepción atomista está estrechamente relacionada con la que hace al modelo de racionalidad subsuntivo. Esta clase de modelo es aquel que entiende al razonamiento moral como la práctica de subsumir hechos en normas. Todo hecho que esté contenido en los supuestos de la norma debe acarrear la consecuencia prevista por ella. Si la norma moral prohíbe mentir, cualquier acto en el que se encuentre la propiedad "no decir la verdad" será moralmente incorrecto. Bajo esta forma del razonamiento moral se obvian propiedades o aspectos destacados (salient features) que dan forma (shape) a la situación concreta ${ }^{18}$. Y es este modelo de donde viene el mote de "legalismo moral".

La crítica de Dancy está dirigida desde lo que él llama "racionalidad narrativa" que entra en contraste con la "racionalidad subsuntiva". Dancy explica esta diferencia con un ejemplo estético. E1 autor compara la situación con la de alguien que trata de describir un edificio desde un punto de vista artístico y no puramente arquitectónico. La última forma de descripción se enfocaría únicamente en cuestiones relativas al material, dimensiones, proporciones y formas, técnicas de construcción, etc., mientras que la primera, tendría que dar cuenta de otros factores destacados como podrían ser ciertos contrastes entre las formas geométricas, las sombras que generan, detalles particulares de las cornisas, etc., que son los que ayudan a que

${ }^{17}$ Martínez Zorrilla, D., Conflictos constitucionales, ponderación e indeterminación normativa, Marcial Pons, Colección Filosofía y Derecho, España, 2007, pág. 169

${ }^{18}$ Dancy, J., Moral... op. cit., pág. 112 
podamos emitir un juicio sobre la belleza o la fealdad del edificio ${ }^{19}$. Martínez Zorrilla nos hace ver que la analogía con la estética, en el caso de Dancy, no es puramente artificial. En realidad, Dancy piensa que el discurso moral es en mayor o en menor medida una percepción más que un razonamiento deductivo; "comprender la situación tiene que ver más con examinar el caso y ver qué elementos destacan y lo configuran valorativamente que con realizar silogismos"20.

Estas dos críticas al universalismo y la forma de configurar el pensamiento moral de Dancy sirven para ver el "dilema de la tolerancia" desde otra perspectiva. Tengamos en cuenta que hemos aceptado la cuestión de que un acto de tolerancia está configurado por tres circunstancias. Dos de ellas requieren, por decirlo con la terminología de Dancy, que tengamos en cuenta los aspectos sobresalientes (salient) que presentan las circunstancias del caso. Me refiero a la primera y a la segunda circunstancia de la tolerancia (la convicción lesionada y la competencia adecuada). Es decir, el agente sujeto de tolerancia tiene que evaluar, primero, si la convicción que ha sido lesionada es moralmente relevante frente al caso en concreto, segundo, tiene que evaluar su calidad jerárquica frente al individuo que la dañó.

Entonces, tras esta perspectiva los casos de tolerancia inician como una forma de razonamiento subsuntivo del tipo: " $\mathrm{X}$ ' está haciendo 'a', de acuerdo con la norma ' $n$ ', 'a' es moralmente incorrecto, por lo tanto, debo condenar la conducta de ' $\mathrm{X}$ '. De acuerdo con la racionalidad subsuntiva, ahí terminaría el razonamiento moral. En el caso de actos de tolerancia, el modelo se complica con algunas variantes más que la alejan del legalismo. El razonamiento en casos de tolerancia podría representarse de la siguiente manera: “' $X$ ' está haciendo ' $a$ ', de acuerdo con mi convicción relevante (norma) ' $n$ ', 'a' es moralmente incorrecto, tengo la competencia necesaria para poder detener la conducta de ' $\mathrm{X}$ ', sin embargo...". Es aquí, en ese "sin embargo", donde se da cuenta de los conflictos normativos generados por la tolerancia. E1 agente de tolerancia repara en la existencia de otra norma contenida

${ }^{19}$ Dancy, J., Moral..., op. cit., pág. 113

${ }^{20}$ Martínez Zorrilla, Conflictos constitucionales..., op. cit., pág. 172. 
en su sistema que le prohíbe aplicar el consecuente de la convicción lesionada. En el texto anterior decía, precisamente, que el dilema de la tolerancia es un dilema in concreto. Esto es, porque las dos normas en pugna pudieron convivir consistentemente dentro de un sistema in abstracto. Sin embargo, hay factores ajenos al razonamiento subsuntivo que hacen llegar al agente moral a ese "sin embargo", a dar cuenta de la incompatibilidad o de la inconsistencia normativa.

En otro lado he explicado que la cuestión de la competencia adecuada no es tan sencilla como el hecho de subsumir nuestra posición en los supuestos contemplados por ciertas reglas (jurídicas, morales, sociales, de etiqueta, etc.) sino que también hay que tomar en cuenta el 'rol' que se estaba jugando en ese momento ${ }^{21}$. Por ello, un amigo puede que padezca el humo de mi cigarrillo mientras nos encontramos en mi oficina platicando, pero que no tolere que fume cuando nos encontramos en su casa; o que mi jefe tolere que hable en una reunión de trabajo, y que lo padezca cuando estamos en el cine. Los factores que pueden darnos la competencia para poder tolerar ciertas conductas son dependientes del contexto donde nos encontremos.

Suele suceder algo similar con la fuerza normativa de las convicciones morales. Puede ser que un padre tolere que su hijo tome coca-cola cuando se encuentran en una fiesta y que no lo haga cuando se encuentran en casa. Las circunstancias pueden cambiar la fuerza normativa de la convicción lesionada. Eso es lo que acarrea, a grandes rasgos, el "sin embargo" que no contemplaba el modelo legalista de racionalidad subsuntiva.

El caso de la madre 'culta' de Vidiella puede reconstruirse en estos términos. Es posible que ella haya permitido, hasta ese momento, que otras personas se volcaran sobre literatura barata, escucharan música "guapachosa" y detestaran los museos de arte contemporáneo. Podemos decir que esta conducta estuvo guiada, entre otras cosas, por la firme convicción de que ella sabía que no contaba con la competencia

${ }^{21}$ González de la Vega, R., "¿Es posible hablar de tolerancia entre iguales? Algunas consideraciones críticas", Diánoia. Revista de Filosofia, LV, 64, 2010, págs. 109-126. 
adecuada para cambiar o interferir en los gustos y en la vida de otras personas, fundamentalmente, frente aquellos relacionados con la falta de sensibilidad estética de algunos individuos; es decir, estaba convencida de que no tenía la competencia moral necesaria para hablar de tolerancia en esos casos. En tal caso, no diríamos que ella toleraba estas situaciones. Podríamos decir que ella era indiferente; aunque dada la relevancia moral que tiene su convicción es algo poco probable. Puede ser que haya sido paciente ante ese hecho. Sin embargo, en el caso de su hija, en primera instancia, ella sí cree tener la competencia necesaria (en su calidad de madre) para poder prohibir que su hija consuma literatura chatarra y música "guapachosa". Empero, recuérdese que el problema en el que se ve inmiscuida es que también sostiene una convicción según la cual es moralmente obligatorio permitir el libre desarrollo de la personalidad de su hija. Convicción, que sin duda, recrudece su situación, pues ella sabe que de intervenir (no tolerar) en las decisiones sustantivas de su hija, puede vulnerar hasta cierto grado el contenido de dicha convicción. El hecho de que se trate de su hija, sin duda, puede considerarse como una razón que "detona" el mecanismo de la tolerancia e incrementa la fuerza normativa de su convicción. Es decir, la madre 'culta' no había sido empujada a pensar en la tolerancia cuando otra persona, ajena a su mundo de personas cercanas, tenía bajos gustos estéticos. Por lo tanto, el asunto sería considerado como el primer caso de lo que podría llegar a ser una serie de casos de (no) tolerancia.

En este sentido, si la madre 'culta' adoptara una postura generalista universalista tendría que optar por una de las dos convicciones en juego: las razones morales que tiene para ser 'culta' y estar en contra de la música guapachosa, de la literatura barata y del escaso interés por los museos de arte o permitir que las personas desarrollen planes de vida de manera autónoma; esto es, que hagan elecciones que estén ausentes de cualquier tipo de compulsión externa.

Sin embargo, como he argumentado hasta el momento, no me parece necesario comprometerse con esta clase de criterios para que la madre 'culta' pueda sostener una decisión moralmente coherente y consistente. En el caso que la acosa, la madre durante años trató de 
inculcarle esta clase de gustos, pudo de alguna forma argumentar que no toleraría que su hija se convirtiera en una amante de la "cumbia" y esto sin que necesariamente implicara una lesión grave a su convicción sobre el libre desarrollo de la personalidad. Puesto que en su rol de madre, ella también consideraba la obligación moral de guiar las elecciones sustantivas de su hija. Por lo tanto, esta decisión, por tratarse de su hija, no la comprometería con el hecho de tener que comenzar a no tolerar al resto de amantes de la 'cumbia' y de los cuartetos cordobeses; no juega el mismo 'rol' que con el resto de los individuos.

La madre debe enfrentarse al hecho de que su hija, ya mayor, se convirtió en contadora, odie la 'poesía' y sea amante de la cumbia y a que ya no tiene competencia sobre su desarrollo y sus elecciones. Ahora bien, aún así puede (como entiendo que lo hizo) hacerle ver a su hija que lo que está haciendo es de mal gusto, que esa literatura o esa música no alimentan el alma ni engrandecen el espíritu; en fin, puede hacerle ver las varias razones que ella tiene para hacerlo. Puede comentarle sobre los beneficios intelectuales del arte y de la buena música, hacerle ver que la buena literatura amplía nuestras perspectivas en la vida, nos engrandece como seres humanos, y nos ayuda a resolver problemas personales, y cualquier otra clase de razones que ella tenga para sostener dicha convicción. Es decir, la madre 'culta' puede fundamentar ante su hijo por qué, ante sus ojos, ella debería hacer el intento de acceder a este mundo estéticamente más sensible y valioso.

Ciertamente, asumir que esta decisión esté moralmente justificada implica reconocer que la madre lo está justificando a través de un juicio moral condicionado. Es decir, que las razones que sustentan dicho juicio moral son relativas a su carácter de madre y que, por lo tanto, no son extensibles a otros casos de características similares. Sin embargo, el hecho de su calidad de madre también funciona como una razón que "detona" su (in)tolerancia y juega el papel de una propiedad relevante que se encuentra ausente en el resto de casos posibles. Esta explicación me parece mucho más plausible que cualquiera de las salidas que hubieran sido propuestas por las posturas generalistas. Las cuales, necesariamente, hubieran obligado a la madre a tener que deshacerse de cualquiera de sus dos convicciones. Lo que, sin duda 
alguna, hubiera representado una pérdida moral mucho más significativa debido a la importancia que les ha otorgado en su vida.

Lo que hasta aquí he argumentado es cómo parte del particularismo de Dancy apoya la idea de la repuesta contextual. La idea general ha sido que, en casos de tolerancia, las circunstancias y los contextos definen el peso específico de cada paquete de razones que tengamos para tolerar o dejar de hacerlo. El problema aquí denunciado trata de reflejar la existencia de una tensión entre los problemas de tolerancia entendidos como conflictos in concreto y los criterios de la universalidad moral.

De todas maneras, la respuesta contextual, tal como la he ido articulando hasta ahora puede ser sometida a la siguiente crítica: hacerme acompañar por Dancy en mis críticas puede hacer pensar que, entonces, estoy decantándome por una postura que niega la idea de que existan principios morales. La cual es una de las tesis centrales de la teoría particularista de Dancy. Si aceptara esta tesis de Dancy, terminaría por abdicar de la objetividad moral que he argumentado tiene la tolerancia, vinculándola con cierta clase de relativismo: el de las circunstancias concretas e irrepetibles, y me alejaría sustancialmente de las partes centrales que me parecen encomiables del liberalismo deontológico.

Sin embargo, esto es salvable diciendo que no acepto completamente el particularismo fuerte de Dancy. Garrett Cullity señala la existencia de dos tesis distintas del particularismo moral: por un lado, encontramos la tesis de que no existen principios morales tal y como suelen ser concebidos por el pensamiento universalista y, por otro lado, está la tesis de que al menos algunas de las circunstancias empíricas de un caso carecen de relevancia moral fija (siempre a favor de su corrección o siempre en contra), sino que estas pueden variar en función del contexto ${ }^{22}$. El particularismo de Dancy defiende conjuntamente ambas tesis, mientras que la tesis que yo defiendo solo recoge la segunda de ellas: la que se podría denominar como particularismo de razones; en contraste con Dancy que defiende tanto un particularismo

${ }^{22}$ Véase, Cullity, G., "Particularism and Moral Theory", en Proceedings of the Aristotelian Society, Vol. 76, págs. 169-90. Y Martínez Zorrila, D., Conflictos constitucionales..., op. cit., p.165-66, nota 37. 
de razones como un particularismo de reglas. De esta manera, sostengo el valor moral de ciertos principios. Sin embargo, pretendo vincular a su actuación normativa una valoración del contexto en el que están siendo invocados. La tesis central de esta postura es que los principios, aún cuando tengan contenidos medianamente determinados, adquieren distinta relevancia moral y fuerza normativa dependiendo de las circunstancias de cada caso en concreto.

Según veo las cosas, la tesis que vengo defendiendo tiene una estrecha vinculación con la idea del residuo moral. Esta idea, como he dejado ver antes, no es bien aceptada por las posturas generalistas universalistas. Recuérdese que hace un momento hablaba de que no tolerar que su hija escuchara cumbia no representaba una lesión grave a su convicción sobre el libre desarrollo de la personalidad. Esto implica que, de todas maneras, la decisión de no tolerar el hecho de que su hija no sea amante de la poesía y del arte implica un sacrificio moral frente a su sistema de valores. Ahora, en lo que sigue me ocuparé de este otro tema como parte de las críticas que tengo ante la postura generalista universalista de la tolerancia.

La última crítica que tengo ante esta perspectiva es que olvida por completo que tras la derogación o la afectación eventual de alguna de las convicciones en conflicto, el agente de tolerancia podría experimentar sensaciones de pérdida moral, sacrificio o remordimiento por la forma en que decidió actuar.

La crítica inicia con la premisa de que las teorías morales generalistas se rigen por un principio rector del sistema (que, en algunos casos, es la autonomía de las personas y, en otros, la felicidad general), el cual es comúnmente considerado como un principio incuestionable. Esta situación, no solo recrudece los conflictos generados por actos de tolerancia y los hace, en muchos casos, imposibles de solventar tal y como he argumentado aquí y en el primer artículo-, sino que también obvia una parte importante de nuestras vidas como agentes morales. Lariguet distingue entre dos clases de residuo moral que pueden generarse al "resolver" conflictos trágicos o dilemáticos como el de la tolerancia: uno es el residuo psicológico, y el otro, el residuo 
normativo ${ }^{23}$. El primero está relacionado con estados mentales como la "culpa", la "vergüenza", el "remordimiento", la "tristeza", etc. En cambio, el residuo normativo se refiere a la existencia de una norma, principio o valor moral que ha sido dejado de lado.

La distinción que hace Lariguet no me parece ociosa desde un punto de vista analítico, sin embargo, creo que en términos generales ambas clases de residuos se solapan. La pérdida que se experimenta por haber violentado una norma o un valor que consideramos relevante tiene expresiones psicológicas como el remordimiento o el sentimiento de culpa; es decir, que la violación de una convicción relevante es la fuente de esas expresiones psicológicas. Por ello, posiblemente, es que autores como Bernard Williams o Martha Nussbaum siempre hagan referencia al residuo como consecuencia psicológica y no normativa, pues piensan que la última presupone a la primera ${ }^{24}$. Aquí lo que me interesa destacar, más allá de esta discusión, es algo muy breve: la indiferencia teórica ante el residuo moral se puede ver, también, como un error de las teorías morales generalistas para dar cuenta de actos de tolerancia.

Típicamente, la tolerancia, se ha visto como el menor de dos males. Sin embargo, esta concepción de la tolerancia ha recibido distintas connotaciones. Una de ellas es aquella que habla de dos males vistos como costos instrumentales, un conflicto entre una acción que puede acarrear peores consecuencias que tomar otro curso de acción (tolerar a los hugonotes puede acarrear beneficios económicos, no tolerarlos puede acarrear beneficios para la paz social). Bajo el esquema de la tolerancia como ideal moral, también se puede ver a la tolerancia como la decisión entre dos males. Pero en este caso, como lo he tratado de argumentar, los dos males a los que se enfrenta el sujeto de tolerancia son acarreados por el fatal incumplimiento de una de las normas morales que están en juego (si tolero que mi hija consuma literatura

${ }^{23}$ Lariguet, G., Encrucijadas Éticas, Una aproximación a los Dilemas y su Impacto en el razonamiento Práctico, Theoria cum Praxi, Madrid/México, 2011, pág. 49 y ss.

${ }^{24}$ Véase, Williams, B., Shame and Necesity, University of California Press, 1993., y Nussbaum, M., El Paisaje del Pensamiento, La inteligencia de las emociones, Paidós, Barcelona, 2008. 
barata y deteste la poesía, estoy permitiendo que se siga aniquilando su sentido estético y mantenga un alma vacía. En cambio, sino no lo tolero, estoy violentando su libertad y su autonomía). El negar la posibilidad de que exista una especie de pérdida o sacrificio moral significa, por lo tanto, que la tolerancia tampoco puede entenderse de esta manera, es decir, como el menor de dos males morales.

El problema nodal de todo esto ha sido denunciado por Bernard Williams en un texto titulado Ethical Consistency. Este problema incluso llevó a Sócrates, en una ocasión, a pensar que algunos dioses sostenían falsas creencias o reclamos injustificados precisamente porque, a veces, los hombres se veían encerrados entre dos reclamos morales ${ }^{25}$. Lo que dice Williams es que el problema se encuentra en querer equiparar los conflictos entre convicciones morales con los conflictos entre creencias. Pero, precisamente, dice Williams, por las características propias de cada uno es mejor no equipararlos. En un conflicto entre creencias, lo más racional es tratar de encontrar cuál de las dos creencias en conflicto es la verdadera. Si una de ellas resulta verdadera, aquí sí, por razones lógicas la otra no lo es y el individuo se verá en la necesidad de desecharla ${ }^{26}$. Sin embargo, en los casos de conflictos morales este no es el caso. Es por ello que estos son más parecidos a los conflictos entre deseos. Si uno de ellos es el vencedor dentro de la deliberación, el otro no necesariamente es abandonado ${ }^{27}$.

El problema que presenta el generalismo moral al no prestar atención a la tesis de los residuos morales puede ser más grande de lo que comúnmente los universalistas argumentan. Es decir, no es suficiente con calificar todas las sensaciones como factores humanos que care-

${ }^{25}$ Nussbaum, M., The Fragility of Goodness. Luck and Ethics in Greek Tragedy and Philosophy, Cambridge, Cambridge University Press, 2001, pág. 30.

${ }^{26}$ Repárese que en estos casos, el desechar una creencia que se tomaba por verdadera después de descubrir que no lo es también puede generar un sentimiento de pérdida. Piénsese en un científico o un historiador que debido a esto pierde una teoría que le parecía correcta y en la que había invertido mucho. Véase, Williams, B., "Ethical Consistency", en Gowans; G.W. (ed.), Moral Dilemmas, Oxford University Press, 1987, pág. 120.

${ }^{27}$ Williams, B., "Ethical Consistency", op. cit., págs. 117-8. 
cen de toda base racional. Con esa "omisión" de sus teorías, lo que están generando es que los agentes sujetos de tolerancia dejen a un lado de su deliberación una situación de suma relevancia para decidir. Pues el mero pronóstico de que mi decisión pudiera acarrear alguna pérdida o sacrificio moral puede lograr que cambie el curso de mi decisión y, por ello, tolerar o no tolerar el acto que me puso en esa situación en un primer término. Bajo los presupuestos del universalismo generalista, se están obviando situaciones en las que decidir entre una de las dos convicciones en conflicto sea imposible o francamente doloroso desde un punto de vista ético (más no desde un punto de vista lógico). Sobre todo, si nos referimos a todos aquellos factores sobresalientes — como la culpa o el remordimiento que se prevé sufrir-que se encuentran en juego.

Tras la lente del universalista se pierden de vista situaciones donde, incluso, alguien pueda elegir un curso de acción que sea consistente con los requerimientos lógicos del sistema, pero que, al mismo tiempo, sea esa acción la que lo ubique como culpable de haber cometido un acto inmoral ${ }^{28}$. Todos estos escenarios, a mi juicio, le presentan a esta perspectiva mayores dificultades que facilidades para poder ejercitarse plenamente frente a la clase de conflictos generados por la tolerancia.

Ahora bien, negarle relevancia práctica a las sensaciones de pérdida o sacrificio hace que las teorías generalistas universalistas pierdan de vista un gran número de consecuencias prácticas que rodean a la tolerancia. Digamos que si el sujeto de tolerancia puede, por cualquier medio, pronosticar cuál será la pérdida o el sacrificio que sufrirá al decantarse por uno de los cuernos del dilema, esta posibilidad puede cambiar el peso de la balanza en su decisión y, por ende, cambiar el curso de su decisión. Con esto en mente, ahora pensemos qué sucedería si que el acto tolerado fuera cometido por alguien que forma parte

${ }^{28}$ El punto central de esta afirmación es que incluso cuando razonamos a través del sistema, llevar a cabo la acción que este nos indica como necesaria no es suficiente para que no seamos culpables o sujetos de responsabilidad moral. Piénsese, por ejemplo, en Agamenón. "En el coro de la obra, el sacrificio de Ifigenia se entiende como algo necesario, sin embargo, en el coro también culpan a Agamenón". Véase, Nussbaum, M., The Fragility..., op. cit., pág. 33. 
del escenario permanente de su vida cotidiana. Es decir, un individuo que se presume juega un rol sumamente importante para el desarrollo de su plan de vida. En estos casos, es presumible que el agente esté dispuesto a suavizar o relativizar una de sus convicciones relevantes, sin tener que deshacerse de ella o sin tener que tolerar un acto similar pero que ahora sea cometido por un individuo ajeno a su círculo de personas relevantes. Tomemos, nuevamente, el ejemplo de la madre 'culta'. En este caso, la madre no guardaría los mismos sentimientos acerca de su convicción si se tratara de otra persona. Sin embargo, el pronóstico de que habrá una pérdida es más fuerte por tratarse de su hija. En otras palabras, y usando otro ejemplo, no es lo mismo aceptar que hay delincuencia en el mundo, que aceptar que tu hijo es el delincuente.

La perspectiva del universalista moral determina que los sujetos de tolerancia no solo tienen que deshacerse de convicciones que les parecen moral o éticamente relevantes (justificadas), sino también desatender cuestiones tan importantes como pueden ser sus planes personales de vida, proyectos intelectuales o profesionales, relaciones sentimentales, entre otras cosas. Me parece que bajo este prisma el costo de la tolerancia es mucho más elevado que el de la intolerancia.

\section{III.}

Dentro del universo de comentarios que me han dirigido, hay una afirmación que me parece ha logrado encapsular mis preocupaciones centrales y que me auxilia para catapultar mis argumentos finales. La última línea del texto de Graciela Vidiella dice: "Después de todo, la pregunta socrática es tan importante como la kantiana". Esta frase, como toda gran frase, resume en pocas palabras la gran batalla que muchos filósofos estamos tratando de pelear y termina por subrayar el quid de la cuestión.

Por ello, no quiero terminar sin ser explícito en algo. Mi crítica al liberalismo deontológico corre por una doble vía: por un lado, intenta hacer una limpieza conceptual y un análisis de compatibilidad práctica y epistémica de todas sus piezas. Me parece que, en ocasiones, los 
liberales seguimos utilizando términos y conceptos por una inercia guiada por la tradición lingüística y conceptual (conceptos y términos que otrora, debido a razones de carácter histórico, eran útiles y servían a propósitos específicos y encajaban en sus estructuras normativas), pero que hoy en día en vez de auxiliar el desarrollo filosófico de esta doctrina, la entumecen o la empantanan, por ser contradictorios con nuevos rasgos teóricos y criterios de razonamiento; claramente sostengo que uno de estos criterios es el de "universalismo generalista", en el caso de los conceptos, guardo mis dudas con el de la "laicidad", por ejemplo ${ }^{29}$. Con esto quiero inferir que una defensa del liberalismo deontológico consiste, también, en depurar su catálogo de figuras normativas y términos conceptuales para mantener una coherencia filosófica.

Por el otro lado, mi crítica coincide con la de otros críticos del liberalismo, como Martha Nussbaum, por ejemplo ${ }^{30}$. Es decir, no pretende dejar atrás al liberalismo deontológico o deshacerse de él (pues, junto con Vidiella, estoy convencido de que en la actualidad es tan necesaria esta perspectiva para dar cuenta de todas las cuestiones relacionadas con los derechos morales y con los principios de justicia, pero también es importante que tome en cuenta otra perspectiva sobre las virtudes y la vida buena), sin embargo, estoy convencido de que el deontologismo liberal ha sido negligente frente a ciertos elementos y aspectos también importantes de la moralidad, como: un desarrollo más detallado del ámbito de lo bueno, el papel de las virtudes morales en la sociedades democráticas o la posibilidad de compaginar una concepción de la vida buena con los rasgos de la imparcialidad requeridos por el deontologismo. Pero del mismo modo considero que para

${ }^{29}$ Consideraría que esta clase de términos y conceptos que todavía guardan un lugar en las filas del pensamiento liberal se mantienen más por razones de carácter emotivo que por su funcionalidad práctica o epistémica.

${ }^{30}$ Véase Nussbaum, M., Political Emotions. Why Love Matters for Justice?, Cambridge, Harvard University Press, 2013; Es en este sentido en el que Nussbaum dice que no pretende ir más allá de Rawls, pero sí cree necesario complementar su teoría de la justicia. 
lograr esto debe dejar atrás otros aspectos como su carácter legalista construido a partir del universalismo generalista.

Basta leer estas líneas para darse cuenta de que, en definitiva, no estoy de acuerdo con la objeción de que estas cuestiones no deben ser atendidas por la filosofía política y moral liberal y, que de hacerlo, se traicionaría nuestra calidad de liberales. Muy por el contrario, considero que en la actualidad esa es la clase de preguntas a las que deberíamos estar dirigiendo nuestros esfuerzos filosóficos y las que deberíamos adoptar como una brújula que marca nuestro Norte filosófico.

\section{Bibliografía}

Cullity, G., "Particularism and Moral Theory", en Proceedings of the Aristotelian Society, Vol. 76.

Dancy, J., Moral Reasons, Oxford \& Cambridge, Blackwell, 1993.

Dancy, J., Ethics Without Principles, Oxford, Clarendon Press, 2006.

González de la Vega, R., "¿Es posible hablar de tolerancia entre iguales? Algunas consideraciones críticas", Diánoia. Revista de Filosofia, LV, 64, 2010.

Hare, R.M., Freedom and Reason, Clarendon Press, Oxford, 1964.

Kant, I., Groundwork of the Metaphysics of Morals, Cambridge, Cambridge University Press, 1997.

Lariguet, G., Encrucijadas Éticas, Una aproximación a los Dilemas y su Impacto en el razonamiento Práctico, Madrid/México, Theoria cum Praxi, 2011.

Luján, J., Palabras manzana, Aique, Buenos Aires, Argentina, 2010.

Martínez Zorrilla, D., Conflictos constitucionales, ponderación e indeterminación normativa, España, Marcial Pons, Colección Filosofía y Derecho, 2007.

McDowell, J., "Non-cognitivism and Rule-following", en Ted Honderich (ed.) Morality and Objectivity: A Tribute to J. L. Mackie, London, Routledge and Kegan Paul, 1985.

Nino, C.S., Ética y Derechos Humanos. Un Ensayo de Fundamentación, Buenos Aires, Astrea, 2005. 
Nussbaum, M.C., El Paisaje del Pensamiento, La inteligencia de las emociones, Barcelona, Paidós, 2008.

Nussbaum, M.C., Political Emotions. Why Love Matters for Justice?, Cambridge, Harvard University Press, 2013.

Nussbaum, M.C., The Fragility of Goodness. Luck and Ethics in Greek Tragedy and Philosophy, Cambridge, Cambridge University Press, 2001.

Wallace, J.D., Moral Relevance and Moral Conflict, Nueva York, Cornell University Press, 1988.

Williams, B., Ethics and the Limits of Philosophy, Cambridge, Harvard University Press, 1985.

Williams, B., "Ethical Consistency", en Christopher W. Gowans (ed.), Moral Dilemmas, Oxford, Oxford University Press, 1987.

Williams, B., Shame and Necessity, California, University of California Press, 1993. 